livraisons

d'Histoire

de l'Architecture

\section{Livraisons de l'histoire de l'architecture}

16 | 2008

Grands chantiers et matériaux

\title{
Reconstruire Vézelay et restaurer la Bourgogne : le choix des matériaux chez E.-E. Viollet-le-Duc
}

Rebuilding Vézelay and restoring in Burgundy: Eugène Viollet-le-Duc's choice of materials

Vézelay wieder aufbauen und Burgund restaurieren : die Auswahl der

Materialien bei Eugène Viollet-le-Duc

\section{Arnaud Timbert}

\section{OpenEdition}

Journals

Édition électronique

URL : https://journals.openedition.org//ha/186

DOI : 10.4000/lha.186

ISSN : 1960-5994

Éditeur

Association Livraisons d'histoire de l'architecture - LHA

Édition imprimée

Date de publication : 10 décembre 2008

Pagination : 87-108

ISSN : 1627-4970

Référence électronique

Arnaud Timbert, «Reconstruire Vézelay et restaurer la Bourgogne : le choix des matériaux chez E.-E. Viollet-le-Duc », Livraisons de l'histoire de l'architecture [En ligne], 16 | 2008, mis en ligne le 10 décembre 2010, consulté le 21 septembre 2021. URL : http://journals.openedition.org//ha/186 ; DOI : https:// doi.org/10.4000//ha.186

Ce document a été généré automatiquement le 21 septembre 2021.

Tous droits réservés à l'Association LHA 


\section{Reconstruire Vézelay et restaurer la Bourgogne : le choix des matériaux chez E.-E. Viollet-le-Duc}

Rebuilding Vézelay and restoring in Burgundy: Eugène Viollet-le-Duc's choice of materials

Vézelay wieder aufbauen und Burgund restaurieren : die Auswahl der

Materialien bei Eugène Viollet-le-Duc

Arnaud Timbert

Depuis plusieurs années diverses études ont été offertes sur Eugène-Emmanuel Viollet-le$\mathrm{Duc}^{1}$; si elles ont favorisé la connaissance du théoricien jusqu'à investir son imaginaire ${ }^{2}$, elles ont néanmoins négligé le praticien. C'est pourquoi, depuis 2006, une réflexion a été engagée sur « Le choix des matériaux chez E.-E. Viollet-le-Duc » à l'École du Louvre et à l'université Charles-de-Gaulle-Lille 3, en partenariat avec le laboratoire de recherches des monuments historiques.

Dans le cadre de ce programme des études ont été effectuées sur des monuments tels que la cathédrale de Clermont-Ferrand, le château de Pierrefonds, la chapelle des catéchismes d'Amiens et l'abbatiale de Saint-Denis ${ }^{3}$ tandis que la Bourgogne était investie dans sa globalité. Cette région a en effet pour intérêt de réunir les trois aspects de Viollet-le-Duc, savoir, le restaurateur, le constructeur, à travers l'église d'Aillant-sur-Tholon (Yonne) notamment ${ }^{4}$, et le théoricien. En témoigne le nombre des exemples bourguignons - en particulier icaunais - du Dictionnaire et des Entretiens.

C'est par ailleurs dans cette région que l'architecte restaurateur fut confronté à une diversité de problèmes sans précédents, liés à l'intervention sur des monuments anciens, pour lesquels des solutions devaient être inventées. L'étude matérielle de ces chantiers, alimentée par des sources abondantes, révèle l'éventail assez large des critères retenus pour la sélection des matériaux, principalement la pierre, le bois et le métal ; ces critères répondent à des soucis archéologiques et architectoniques, à des impératifs économiques 
autant qu'à des choix esthétiques, sans qu'il soit toujours possible de discerner la part revenant à l'un ou à l'autre non plus qu'une logique d'ensemble.

\section{Les sources}

4 Durant toute sa carrière, la Bourgogne est la région qui occupa Viollet-le-Duc le plus régulièrement, comme l'indiquent ses nombreux déplacements pour inspection ${ }^{5}$ ou à l'occasion de rapports sollicités par son administration ${ }^{6}$. À partir de 1840 , avec la restauration de la Madeleine de Vézelay, qui inaugure les travaux de la Commission des Monuments historiques, l'architecte engage plusieurs chantiers. Ainsi, débutent dès 1842 la rénovation de Saint-Andoche de Saulieu (1842-1846) ${ }^{7}$, de Semur-en-Auxois (1843-1854) ${ }^{8}$, de Flavigny-sur-Ozerain (1844-1847) ${ }^{9}$ et de la collégiale de Beaune (1844-1860) ${ }^{10}$. En 1845 ses efforts se concentrent dans l'Yonne avec la reprise des vitraux de Saint-Julien-duSault (1843-1854) ${ }^{11}$. Il dirige par ailleurs la réfection de la crypte de la cathédrale SaintEtienne, à Auxerre $(1845-1848)^{12}$, et celle de la galerie romane du palais épiscopal (1845-1846) où il érige une cuisine en style néo-gothique (1847-1848) ${ }^{13}$. Toujours en 1845 , il entreprend la restauration des églises de Saint-Thibault-en-Auxois ${ }^{14}$, de Saint-Pèresous-Vézelay ${ }^{15}$ et de Montréal (1845-1852) ${ }^{16}$; en 1847, il est en charge de la porte SaintAndré d'Autun ${ }^{17}$ tandis que de 1855 à 1866 il réhabilite la salle synodale de Sens ${ }^{18}$ et construit l'église d'Aillant-sur-Tholon (1865-1867) ${ }^{19}$.

5 L'organisation de ces chantiers est particulièrement bien éclairée par la correspondance échangée entre Viollet-le-Duc et ses collaborateurs. La première, avec François-Nicolas Comynet, inspecteur des travaux de la Madeleine de Vézelay de 1840 à 1846, est riche sur le sujet, mais n'intéresse que ce monument ${ }^{20}$; en revanche, celle entretenue avec Émile Amé ${ }^{1}$, inspecteur en charge de la Bourgogne, bien que moins prolixe en détails techniques, embrasse tous les chantiers en cours de 1846 à $1857^{22}$. Il faut également citer le fonds de lettres de Viollet-le-Duc au maire d'Aillant-sur-Tholon ${ }^{23}$; il nous informe sur un chantier mené à l'économie ${ }^{24}$.

6 Les Rapports préalables aux travaux comme les Mémoires en résultant restent toutefois les sources les plus riches sur le sujet. Ces documents, destinés à la commission des monuments historiques, permettent d'apprécier la multiplicité des critères qui présidèrent aux choix des matériaux, tout en nous informant sur la démarche prospective et les méthodes constructives d'Eugène-Emmanuel Viollet-le-Duc ${ }^{25}$.

\section{La pierre}

7 À Vézelay et dans les monuments bourguignons, le choix des carrières repose sur des arguments archéologiques élaborés dans la perspective de garder l'unité géologique des édifices restaurés. Il repose également sur des critères techniques à valeur architectonique afin d'utiliser la pierre selon une densité déterminée par sa destination ; sur des impératifs économiques permettant de construire, mais également de restaurer, à moindre frais, et sur une orientation esthétique destinée à sauvegarder l'harmonie des couleurs lithiques.

Des considérations d'ordre archéologique : dès mars 1840, dans son rapport adressé à la Commission des Monuments historiques au sujet de la Madeleine de Vézelay, Viollet-leDuc propose une identification géologique des pierres mises en œuvre au XII ${ }^{\mathrm{e}}$ siècle : «J'ai 
dû pendant mon séjour à Vézelay rechercher quels étaient les matériaux qui avaient servi à la construction de l'église de la Madeleine afin d'employer les mêmes s'il est possible dans les travaux de restauration. » Aussi l'architecte observe-t-il sept types de pierre dont il s'efforce de localiser les carrières: «Une pierre grise avec grandes tâches jaunâtres, grès, carrière inconnue. Une pierre dure grossière ... grès je crois, carrière [sic] proches Vézelay encore ouverte aujourd'hui. Une pierre moins dure que la précédente, calcaire assez fin, carrières dites de la Mance encore exploitées. 3 lieues de Vézelay. Une pierre très blanche, calcaire, carrière inconnue. Une pierre blanche jaunâtre assez dure, calcaire recevant le poli, carrière de Coutarnoux, encore exploitées, 7 lieues de Vézelay. Une pierre blanche assez jaunâtre, très fine, calcaire, c'est avec cette pierre que sont faites les colonnes monolithes du chœur. Je crois que cette pierre vient de Tonnerre ${ }^{26}$. Moellon calcaire se délitant facilement, appelé lave dans le pays. Extrait autour de Vézelay ${ }^{27}$. "

Viollet-le-Duc identifie donc, avec plus ou moins de difficultés ${ }^{28}$, la nature des matériaux et recherche les carrières d'origine afin de les exploiter ${ }^{29}$. Cette démarche, qu'il réitéra plus tard à Autun - pour la porte Saint-André ${ }^{30}$ - et à Pierrefonds ${ }^{31}$, illustre la volonté de préserver l'authenticité matérielle du monument.

matériaux autorise la même remarque. L'architecte invite en effet ses tailleurs de pierre à faire le plus grand cas du layage. La densité de la pierre détermine son outil ${ }^{32}$, « il faut [écrit-il à Émile Amé] que tout ce qui est du XIII ${ }^{e}$ siècle soit taillé à la bretture ${ }^{33}$ » et lui suggère de "faire attention aux matériaux de maçonnerie [...] ils sont souvent trop grossièrement layés ${ }^{34} »$. La majorité des assises neuves sont ainsi travaillées au taillant droit dans les parties romanes de la Madeleine tandis que la gradine et le taillant bretté sont réservés à l'œuvre gothique du chevet ${ }^{35}$. Seul écart avec la technicité de la taille médiévale, dans les deux cas, l'apport d'une large ciselure périmétrique, d'inspiration néo-classique et inhérente à la formation du XIXe siècle, est étrangère à l'édifice. Au-delà de ces recommandations sur les outils, l'architecte sollicite la réalisation de marques lapidaires pour les assises neuves de telle sorte à ne pas rompre avec l'abondante glyptique médiévale de la Madeleine. Cette démarche dévoile ainsi un souci aussi archéologique qu'esthétique du choix et du traitement de la pierre ${ }^{36}$.

11 Toutefois, c'est le plus souvent la résistance des matériaux à disposition qui détermine Viollet-le-Duc.

Dans son rapport de 1840, il fait une distinction entre la pierre de Coutarnoux et celle de la Mance ainsi que, plus tard, entre la pierre de la Mance mi-tendre et la pierre de la Mance dure ${ }^{37}$, l'obligeant à un choix nuancé lors de la mise en œuvre du cloître de Vézelay, de l'avant-nef et de son escalier ${ }^{38}$. Pour ce dernier, la pierre de Coutarnoux, tendre, est choisie pour les arcs, la pierre de la Mance, mi-tendre, pour les sommiers, et la pierre de la Mance, dure, pour les supports (ill.1). 


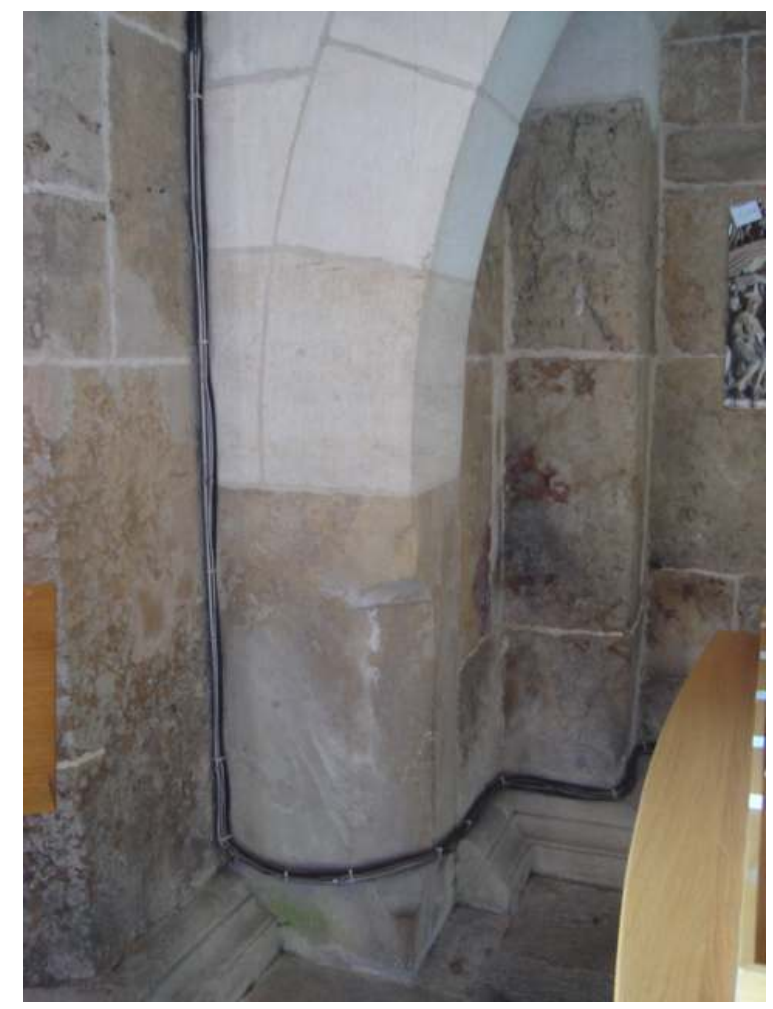

Support et sommier de l'escalier de l'avant-nef. Détail

Cl. A.Timbert

Dans le même ordre, à l'église d'Aillant-sur-Tholon, la pierre dense et non gélive de Chevroches ${ }^{39}$ est employée pour les soubassements de l'édifice tandis que celle, plus tendre, de Molesmes, est réservée aux contreforts, aux tableaux des baies et aux corniches. À l'intérieur, une distinction s'impose entre calcaire dense en pierre de Chevroches pour les parties inférieures des piles et calcaire tendre, type pierre de Tonnerre, pour les parties supérieures (ill.2) ${ }^{40}$. La même répartition s'observe à la salle synodale de Sens où la pierre de Chevroches est réservée, entre autres, aux contreforts, aux chéneaux et aux gargouilles, quand celle de Molesmes est limitée au décor sculpté et aux colonnettes ${ }^{41}$. Rien de particulièrement étonnant en somme : Viollet-le-Duc fait un choix et un usage pertinents des matériaux selon leur destination dans le monument, fort d'un savoir-faire assimilé de longue date par la corporation ${ }^{42}$. 
III. 2: Aillant-sur-Tholon,

église Saint-Martin.

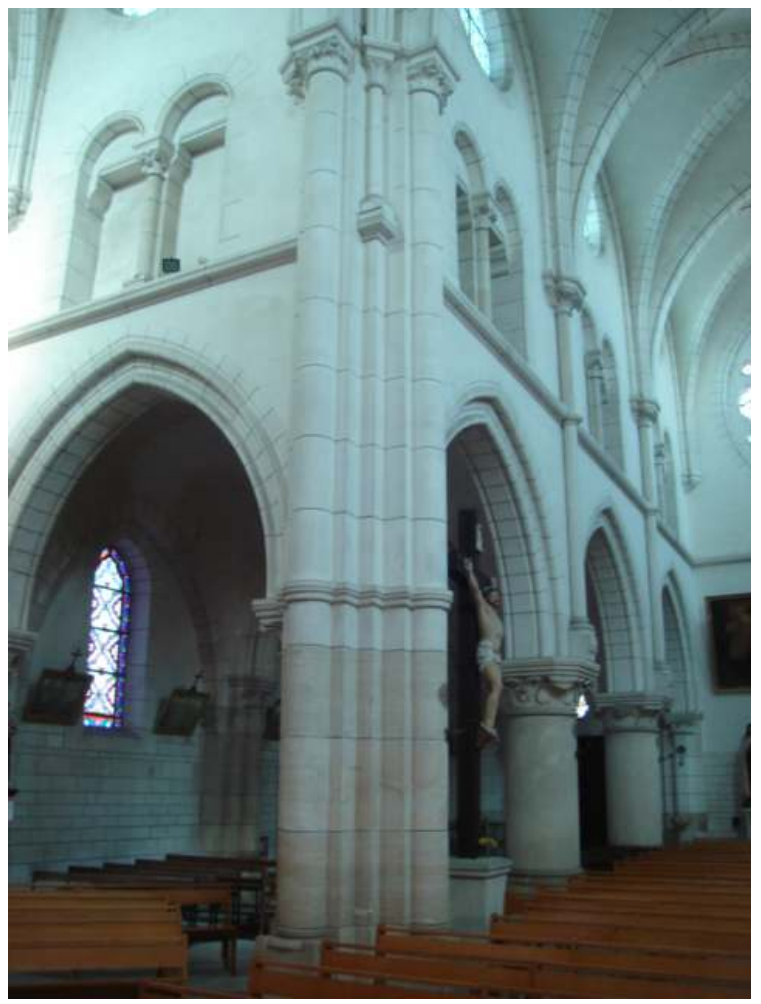

Support nord-ouest de la croisée du transept

Cl. A.Timbert

À Vézelay, d'après l'évaluation réalisée sur le fondement des mémoires ${ }^{43}$, la pierre de la Mance est de loin la plus utilisée (environ $2313 \mathrm{~m}^{3}$ ), notamment pour la reconstruction des arcs-boutants et des contreforts de la nef ainsi que pour les parties hautes de l'avantnef et son pilier sud-ouest. La pierre de lave ${ }^{44}$ a servi à la réfection des voûtes tandis que celle de Coutarnoux (environ $\left.900 \mathrm{~m}^{3}\right)^{45}$ a été adoptée pour la sculpture, pour les parois intérieures du chevet et les volées de ses arcs-boutants. La pierre de Courson ${ }^{46}$ est réservée aux arcs doubleaux du haut vaisseau; enfin, la pierre de Thizy ${ }^{47}$, au même titre que certaines "pierres de l'administration", constitue le dallage ${ }^{48}$. Nous pouvons observer un usage identique de ces matériaux pour les autres monuments restaurés par Viollet-le-Duc en Bourgogne. Outre quelques spécificités locales avec la pierre de Montbard, employée à Semur et à ,Saint-Thibaut-en-Auxois ${ }^{49}$, celle de Meursault à Beaune ${ }^{50}$ et de Produns à Autun ${ }^{51}$, la pierre de Coutarnoux est choisie pour les piles, les contreforts et les réseaux de la rose de l'église de Montréal $^{52}$; la pierre de Courson est retenue pour les parements et le décor sculpté de la galerie romane d'Auxerre ${ }^{53}$; celle la Mance pour les arcs-boutants de la nef de Saint-Père-sous-Vézelay ${ }^{54}$ tandis que la pierre de Thizy est utilisée en dallage à Montréal ${ }^{55}$ et à Sens ${ }^{56}$. Pour cette dernière fonction, la pierre d'Anstrude (calcaire oolithique, Jurassique moyen, Bathonien) extraite à Bierryles-Belles-Fontaines (Yonne) a souvent la faveur de Viollet-le-Duc. Elle est utilisée à Semur-en-Auxois (1843-1854) pour le pavage, les contreforts et les parties hautes de la flèche ${ }^{57}$. Il l'emploie ensuite dans d'autres chantiers tels qu'Aillant-sur-Tholon ${ }^{58}$. Dans ce dernier édifice il envisage toutefois son remplacement par la pierre de Gigny (Côte-d'Or), plus résistante et économique:»En rédigeant le devis pour la construction de l'église d'Aillant, nous avons toujours eu en vue l'emploi des matériaux les plus rapprochés de la localité ou les plus faciles à y faire arriver soit par la rivière d'Yonne, les canneaux ou les 
voies ferrées qui offrent une grande économie sur les transports par voiture. Depuis cette époque (1862) l'embranchement du chemin de fer de Paris à Lyon qui relie actuellement Châtillon à la ligne principale en aboutissant à Nuits-sous-Ravière vient d'être mis en activité et permet d'employer à un prix relativement peu élevé la pierre de Gigny, arrondissement de Tonnerre (Yonne). Cette pierre d'une dureté excessive et d'une belle teinte est de beaucoup supérieure en dureté et en qualité à la pierre d'Anstrude proposée au devis ${ }^{59}$.»

Cette première analyse autorise plusieurs remarques. Viollet-le-Duc utilise un nombre limité et identique de pierres pour l'ensemble des monuments dont il a la charge en Bourgogne, soit dix types, ce qui est relativement modeste comparativement à la seule église de Saint-Denis-de-L'Estrée (13 types) ou du château de Pierrefonds (11) ${ }^{60}$. Par ailleurs, il n'a pas eu recours à des carrières extérieures à la région, ce qui ne sera pas le cas pour ses chantiers parisiens ${ }^{61}$. Enfin, le critère économique est relativement prédominant, qu'il s'agisse des monuments restaurés ou construits, mais il reste néanmoins souvent associé, comme le révèle l'exemple d'Aillant-sur-Tholon, à des arguments techniques: si la pierre de Gigny eut la faveur sur celle d'Anstrude, c'est qu'elle lui paraissait aussi et surtout, plus résistante pour le pavement.

Il convient néanmoins de pondérer cette dernière observation: parfois, la volonté d'employer des matériaux garants de l'authenticité géologique et de la pérennité des monuments disparaît au profit de choix strictement économiques.

Ainsi, à la Madeleine de Vézelay, Viollet-le-Duc opte pour le grand appareil dans la reprise du parement de la première travée sud de l'avant-nef et pour les piédroits de la porte du cloître. Il refuse ainsi le moellon utilisé dans les parties basses du monument, privilégiant la mise en œuvre rapide et économique que favorisent les grands modules, les moyens de transport et de levage de son époque ${ }^{62}$. Le même constat s'impose dès lors qu'il met en œuvre des matériaux étrangers aux monuments restaurés, tels que la pierre de Courson. Pour la reprise des arcs-doubleaux de la nef, il souhaita retrouver, afin de reconstituer l'alternance si caractéristique des claveaux, la pierre rousse communément attribuée aux carrières de Tharoiseau (ill.3) ${ }^{63}$; celles-ci étaient néanmoins épuisées depuis le Moyen Âge. 




Élévation du haut vaisseau

Cl. A.Timbert

Viollet-le-Duc a donc choisi, comme l'indique le devis de 1840, la pierre de la Mance, tout à fait favorable, par sa densité, à ce type de construction ${ }^{64}$. Il revint néanmoins sur ce choix au profit de la pierre de Courson extraite autour de Courson-les-Carrières (Yonne) et assez tendre pour se débiter à la scie ${ }^{65}$; or, cette pierre est immédiatement apparue d'une résistance trop modeste pour une telle destination ${ }^{66}$. De même, malgré le souhait de l'employer pour la sculpture ${ }^{67}$, elle fut considérée comme un matériau " trop plâtreux » à cet effet $^{68}$. Le choix de la pierre de Courson semble ainsi résulter du seul critère économique. Le 19 juillet 1840, Comynet informe Viollet-le-Duc du coût modique de cette pierre: "Vous voyez par le prix que j'ai obtenu qu'elle [la pierre de Courson] est bien moins coûteuse que celle de la Mance puisque celle-ci coûterait 56,02 tandis que celle de Courson ne coûtera que 54 frcs 75 c. $^{69}$ " Ce à quoi Viollet-le-Duc répond: " Vous pourrez dire à $\mathrm{M}$. Demay [l'entrepreneur en maçonnerie] qu'il peut toujours prendre ses mesures pour s'approvisionner de pierre de la Mance et de la pierre de Courson nous emploierons cette dernière pour la reconstruction des arcs doubleaux ${ }^{70}$. " À une pierre dense, idéale pour la réalisation des arcs, Viollet-le-Duc préféra une pierre tendre, moins favorable à la pérennité de l'œuvre, mais peu coûteuse; rapidement les claveaux, trop fortement soumis les uns aux autres, s'écrasèrent et suscitèrent une nouvelle restauration en $1965^{71}$.

Par ailleurs, l'architecte a systématiquement remplacé toutes les couvertures en lave, trop lourdes selon lui, mais en réalité beaucoup trop coûteuses pour son époque ${ }^{72}$; ainsi à Montréal $^{73}$, à Flavigny-sur-Ozerain ${ }^{74}$, à Saint-Père-sous-Vézelay ${ }^{75}$ et à Beaune ${ }^{76}$ les toitures en lave, si caractéristiques des monuments bourguignons, furent détruites au profit de la tuile creuse jusque-là quasiment absente du paysage, tout du moins du paysage icaunais. 
C'est une réflexion du même ordre sur les coûts qui l'invite, dès 1840, à proposer, pour les bas-côtés de la nef de la Madeleine, une couverture en zinc à la fois plus économique que le plomb et le cuivre ${ }^{77}$ et permettant une mise en œuvre rapide comparativement à la tuile ${ }^{78}$. Cette proposition, rejetée par la Commission ${ }^{79}$, témoigne du penchant bien connu de Viollet-le-Duc pour les matériaux économiques que lui offre l'industrie contemporaine - il utilisera d'ailleurs le zinc pour les flèches de Clermont ${ }^{80}$ - et la certitude que les maitres du Moyen Âge les auraient employés s'ils les avaient eus à disposition ${ }^{81}$. Pourtant, dans la même région et à quelques années près, il s'est évertué à restituer, dans une démarche plus archéologique qu'économique cette fois-ci, la couverture en tuiles vernissée de la salle synodale de Sens ${ }^{82}$.

En somme, il n'y a pas de règles strictes, mais des démarches et des méthodes qui se modulent selon les monuments. Une seule constante émerge toutefois de la diversité : le critère économique, intimement lié à l'usage de matériaux modernes. Quelle que soit la nature du choix, qu'il soit d'ordre archéologique, architectonique ou autre, il réapparaît toujours, latent dans la réflexion de l'architecte.

À cet égard, les matériaux qu'il emploie pour le voûtement sont révélateurs. Il utilise la brique pour les voûtes d'Aillant-sur-Tholon, pour des raisons économiques sur lesquelles il s'expliqua à plusieurs reprises, notamment à travers le rapport Duban ${ }^{83}$. De même, à l'église de Saint-Florentin (Yonne), dans un monument historique cette fois-ci, il invite l'architecte Gervais-Julien Piéplu à à substituer la brique à la pierre pour les voûtains ${ }^{84}$. À Sens, il n'agit pas autrement au premier étage de la salle synodale où « les remplissages des voûtes seront faits en poteries hourdées en mortier hydraulique pour ne point charger les vieilles constructions » (ill. 4$)^{85}$.

\section{4 : Saint-Denis, abbatiale. Poterie cylindrique provenant des restaurations du XIX ${ }^{e}$ siècle}

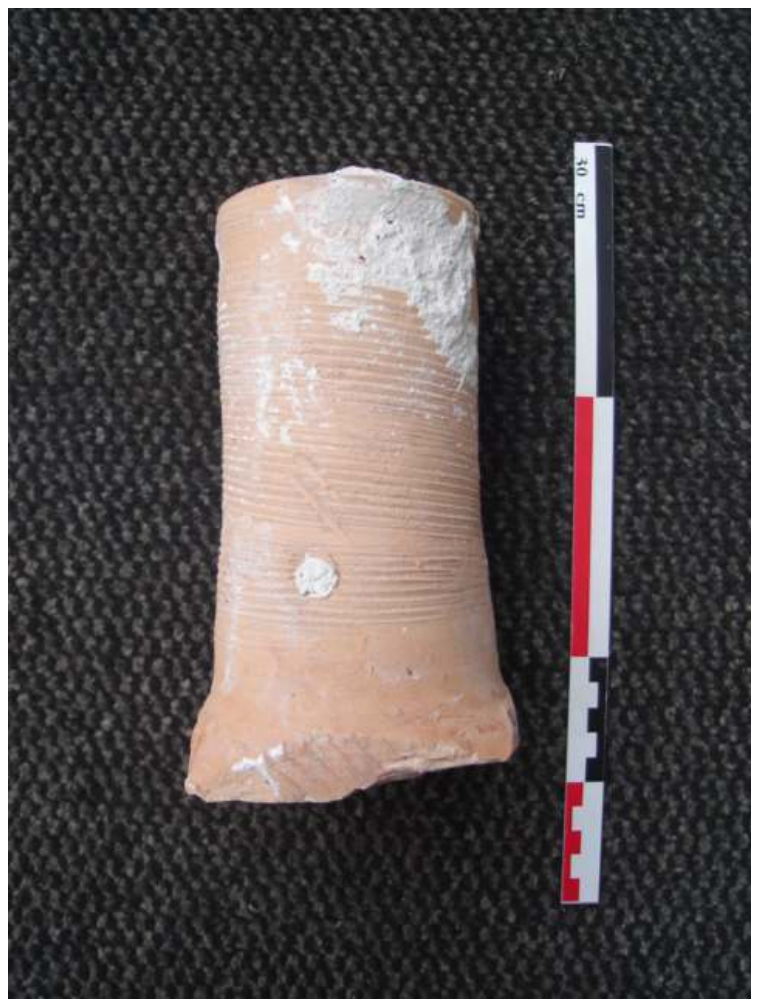

Celles utilisées à Sens devaient être identiques

Cl. A.Timbert 
22 Au-delà des matériaux, les techniques sont également novatrices comme l'illustre un recours à la silicatisation à Amiens et à Saint-Denis ${ }^{86}$, mais également l'usage, dans des proportions inattendues, du plâtre pour la mise en œuvre et le liaisonnement des maçonneries ${ }^{87}$ ou encore celui de l'asphalte, à Notre-Dame de Paris ${ }^{88}$.

À travers le seul choix des matériaux et des techniques déployées pour les traiter ou les mettre en œuvre, il apparaît combien Viollet-le-Duc hésite entre la volonté de restaurer et de prolonger, entre le désir de l'expérimentation archéologique et le souci, constant, de bâtir à l'économie avec des matériaux contemporains, entre l'envie d'être à la fois du Moyen Âge et $\mathrm{du} \mathrm{XIX}^{\mathrm{e}}$ siècle, à la fois, pour reprendre sa propre expression, des morts et des vivants ${ }^{89}$.

Bien qu'il ne se soit jamais prononcé sur le sujet, un dernier critère de sélection des matériaux lithiques, d'ordre esthétique celui-ci, semble avoir été déterminant pour Viollet-le-Duc.

À la Madeleine de Vézelay, certains estiment que les choix de la pierre de Coutarnoux, pour la sculpture, et de la Mance, pour la construction, « se révèlent l'une et l'autre bien décevantes, d'une couleur blanche tranchant violemment dans l'harmonie raffinée de tout l'édifice et imperméables à toute patine ${ }^{90} »$. Ce jugement invite à une légère nuance. D'une part, nous ne savons pas quels étaient les matériaux des arcs-boutants du XII ${ }^{\mathrm{e}}$ siècle avant leur reconstruction ${ }^{91}$; rien n'indique qu'ils n'aient pas été bâtis en pierre de la Mance. Par ailleurs, cette pierre prenant la teinte d'un gris prononcé, lorsqu'elle est mise en œuvre à l'extérieur, si elle rompt avec l'esthétique chatoyante de la pierre de Tharoiseau (ill. 5), reste néanmoins plus discrète que la pierre de Tonnerre, utilisée dans les années $1950^{92}$; elle tâche l'appareil médiéval (ill. 6). 
III. 5 : Vézelay, abbatiale de la Madeleine



Élévation extérieure de la nef, flanc sud. Travée orientale restaurée par Viollet-le-Duc Cl. A.Timbert 


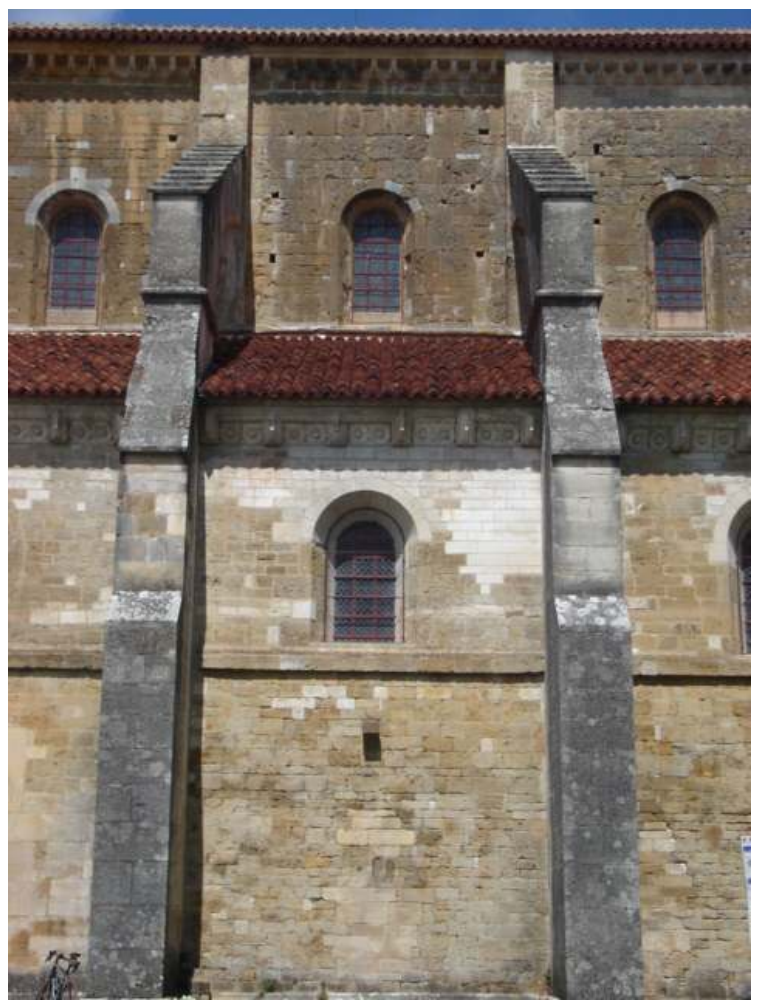

Élévation extérieure de la nef, flanc sud. Travée orientale restaurée par Vassas Cl. A.Timbert.

À l'intérieur, Viollet-le-Duc a su, parfois, employer les bancs les plus ocres de la pierre de La Mance pour ne pas briser le rapport de couleur avec les parements roux du XII ${ }^{e}$ siècle ; de même, il a choisi les bancs les plus clairs de Coutarnoux pour la restauration des supports du chevet, de telle sorte que les reprises ne s'observent qu'à la seule lumière des attachements figurés. Dans le même esprit, afin de ne pas rompre l'alternance chromatique des claveaux composant les arcs doubleaux du haut vaisseau, reconstruits en pierre blanche de Courson, il fit peindre un claveau sur deux en ocre rouge (ill. 3) ${ }^{93}$. Si l'unité matérielle est rompue, l'homogénéité esthétique est préservée.

Viollet-le-Duc procéda de même à Pierrefonds : au commencement de la restauration du château, les parties neuves étaient teintées pour se confondre aux appareils anciens à l'aide de «noir pour noircir les ravalements ${ }^{94}$ ». À Amiens, pour la construction de la chapelle des catéchismes, il réalisa « une superposition plus esthétique que rationnelle des matériaux : les murs gouttereaux, en vergelès de Saint-Maximin, sont élevés sur un soubassement de trois assises de grès surmontées de deux épaisses assises de craie intercalant une pierre tendre entre deux autres de plus forte densité et résistance ${ }^{95}$ » (ill.7); or, Viollet-le-Duc et ses contemporains savaient que la superposition de deux parements de densité différentes contribuait à accélérer le processus de dégradation de la plus tendre ${ }^{96}$. 
III. 7 : Amiens, chapelle des catéchismes

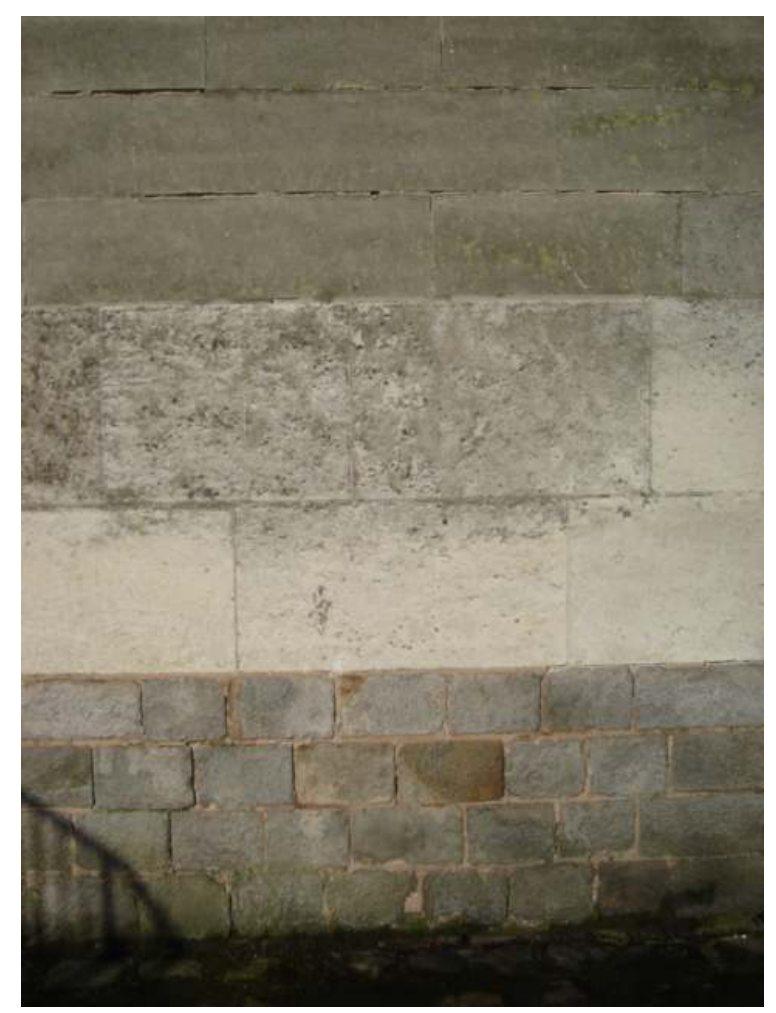

Soubassement. Détail

Cl. A.Timbert

Ici l'esthétique l'emporte sur le rationalisme géologique, il a privilégié le jeu des couleurs naturelles afin de mettre sa construction en harmonie avec le palais épiscopal du XVIII ${ }^{\mathrm{e}}$ siècle. Cette alternance est en effet absente du flanc sud de la chapelle, non mitoyen du palais.

Notons enfin que ce souci d'associer l'ancien au nouveau justifie également, au-delà de critères économiques évidents, le remploi des matériaux. À la cathédrale d'Amiens, « les entrepreneurs seront expressément tenus d'employer, suivant les indications de l'architecte, les matériaux provenant des démolitions pour raccorder les anciennes constructions avec les nouvelles ${ }^{97}$ ». Ce critère de choix d'intérêt esthétique n'est pas absent des chantiers bourguignons. Ainsi, entre autres exemples ${ }^{98}$, écrit-il à Émile Amé au sujet de Montréal : » Je tiens essentiellement à ce qu'on emploi le plus possible des vieux morceaux, non pas tant par économie que par le prix de [ces trois mots barrés] respect pour ces vieilles pierres ${ }^{99}$ » et ce n'est pas moins de $2273 \mathrm{~m}^{3}$ de « vieilles pierres » qui furent remployées à Vézelay ${ }^{100}$. Viollet-le-Duc est ici dans une logique médiévale. À l'instar des maîtres des $\mathrm{XII}^{\mathrm{e}}$ et $\mathrm{XIII}^{\mathrm{e}}$ siècles, il ne conçoit pas la restauration sans assimilation ou absorption matérielle de l'édifice antérieur dans l'œuvre nouvelle. Cette démarche, tout autant que la recherche du matériau d'origine, légitime ses travaux en les inscrivant dans la continuité historique. 


\section{Le bois} bois sec tant pour les cintres que pour la charpente ${ }^{101}$. Toutefois, la situation de ce
chantier est particulière; dès l'annonce de son ouverture les entrepreneurs en charpenterie ont acheté les bois nécessaires aux travaux dans la perspective de les revendre avec une forte plus-value à l'État ${ }^{102}$. Comynet et Viollet-le-Duc se sont donc résolus à employer du bois sec accompagné de bois vert ainsi que du chêne associé à du sapin $^{103}$. Les cintres ont été montés selon une répartition rigoureuse des essences et des qualités : les moises simples en sapin et les moises doubles en chêne, tandis que les bois verts étaient réservés « aux gros poteaux placés debout et qui soutiennent le système de cintrage $^{104} »$. D'une manière générale le sapin et le chêne, auquel il faut parfois ajouter le peuplier, ont eu la faveur de Viollet-le-Duc. Ainsi, comme à Vézelay, le sapin a été retenu pour les échafaudages à Semur-en-Auxois ${ }^{105}$ et à Saint-Thibault-en-Auxois ${ }^{106}$, tandis que le chêne était privilégié pour ceux de Montréal ${ }^{107}$ et la réfection des charpentes de Flavignysur-Ozerain $^{108}$, de Beaune ${ }^{109}$ et de Sens ${ }^{110}$.

La fourniture de ce matériau reste néanmoins d'un coût élevé sur les chantiers bourguignons (60 francs le stère en moyenne) ${ }^{111}$. Aussi la volonté d'en faire un emploi économique invita Viollet-le-Duc à certaines recommandations pour le chantier de Montréal : » Il m'est impossible d'admettre $1 / 5$ de déchet pour les bois, je ne veux pas en entendre parler, il faut que la balance entre le cube brut et le cube employé ne soit pas plus d' $1 / 8^{\text {e }}$ en sus ; cela est déjà énorme, prenez le cube employé et n'admettez donc le mémoire que $1 / 8^{\mathrm{e}}$ en sus pour le bois brut. Il faudra me représenter le reste du bois ou me donner des raisons bonnes, mais très bonnes ${ }^{112}$. " Trois ans plus tard : " Pour Montréale [sic] le devis des bois employés ne doit pas excéder $1 / 5^{\mathrm{e}}$ du bois en grume ou pour être plus clair, le cube du bois mis en œuvre ne peut être moindre des $4 / 5^{\mathrm{e}}$ du bois reçu en grume. Basez-vous là-dessus, la perte qu'on nous a fait éprouver, cette perte devra être compensé [sic] par la suppression d'un ou plusieurs articles du dernier mémoire montant au total de cette perte ${ }^{113}$. $"$ De tels problèmes de gaspillage engendrent par ailleurs des ordres précis sur l'emploi des bois : « Ne faites acheter de peuplier que juste ce qu'il faut, il me semble qu'en faisant refendre un peuplier en deux et en coupant les morceaux de la longueur qui vous est nécessaire, c'est tout ce qu'il faut. Mais je vous prie, pas d'excès de bois puisque nous ne saurions qu'en faire. Il me paraît même qu'avec les vieux bois qui nous resteraient on aurait pu faire l'affaire. Ces travaux de Montréale sont et paraissent déjà bien assez chers ${ }^{114}$. "

Pour amoindrir le coût résultant de l'emploi de ce matériau, lorsque les échafaudages ne sont pas loués, comme ce fut notamment le cas à Sens ${ }^{115}$, le remploi a la faveur de Violletle-Duc. Ainsi, pour la construction de la charpente de l'église d'Aillant-sur-Tholon les bois en bon état de l'église antérieure sont employés pour la nouvelle ${ }^{116}$. À Montréal et à Vézelay, la restauration des combles s'effectue en partie avec le bois des cintres ${ }^{117}$; de même, à Semur-en-Auxois, les combles des chapelles nord du chœur sont rénovés avec le bois de l'échafaudage en sapin ayant servi à la restauration de la flèche ${ }^{118}$.

Pour un usage à l'économie du bois, Viollet-le-Duc propose également diverses solutions parmi lesquelles la formule hybride de la charpente à bois et métal. Ce type de structure permet à la fois l'absence de l'entrait de bois - pièce coûteuse - au profit de l'entrait en fer et l'usage d'un poinçon court. Ce type de charpente a été employé à l'église d'Aillant- 
sur-Tholon, dans la nef de l'abbatiale de Vézelay et dans celle de Beaune au sujet de laquelle l'architecte écrit : " Les grandes voûtes dépassant le niveau des corniches, pour éviter la poussée de cette charpente, dont les entraits ne pourraient être que retroussés, j'ai combiné un système de tirants en fer qui donneraient aux fermes toute la solidité désirable, et empêcheraient totalement la poussée : ce moyen qui du reste n'est pas neuf et qui a déjà été employé avec succès, a de plus, l'avantage d'être économique ${ }^{119}$. " Le métal n'est donc pas un matériau absent des chantiers viollet-le-duciens.

\section{Le métal} goujons pour liaisonner des colonnes à leurs bases ou à leurs chapiteaux, de même que pour consolider l'appareil d'une façade, notamment à Montréal et à la porte Saint-André d'Autun. Pour celle-ci la démarche archéologique justifie le recours au fer : " Les pierres seraient seulement reliées entre-elles par des crampons et goujons en fer semblables à ceux que l'on retrouve dans toutes les constructions romaines ${ }^{124}$. " Le plomb apparaît également, soit sous forme de gangue isolante, entre la pierre et le fer, à Flavigny-surOzerain notamment ${ }^{125}$, soit, ce qui est peut-être plus révélateur d'un regard attentif aux monuments du Moyen Âge, comme matériau de liaisonnement pour les roses de l'église de Montréal ${ }^{126}$ et pour le scellement des étriers des charpentes de Semur-en-Auxois ${ }^{127}$. Si le plomb est, par ailleurs, employé pour la couverture et les noues - voir l'exemple de Saint-Père-sous-Vézelay -, à Saint-Thibaut-en-Auxois, Viollet-le-Duc proposa la réalisation d'une faîtière en fonte peinte ${ }^{128}$; choix étonnant de la part de celui qui s'éleva, dans les mêmes années, contre le faîtage en fonte de Debret à Saint-Denis ${ }^{129}$.

Ce rapide état de nos recherches en cours dévoile les hésitations d'une pensée évolutive et d'une méthode qui se cherche. Quelle que soit la matière envisagée, Viollet-le-Duc hésite constamment entre le désir de restaurer avec des matériaux contemporains, de faire œuvre d'archéologue, de choisir les pierres de telle sorte qu'elles fussent en harmonie de couleur avec les parements anciens et de travailler avec la plus grande économie. Si ce dernier critère apparaît toujours en filigrane, aucun des précédents ne prédominent; ils cohabitent dans l'esprit de l'architecte avec une apparente confusion 
qu'il est difficile d'éclaircir et si parfois l'un semble avoir sa préférence sur l'autre, il s'en explique, se justifie auprès de ses inspecteurs autant de fois que le nécessite l'absence de règles. L'absence de règles, en effet; bien qu'il y ait déjà, dans ce second quart du XIX ${ }^{e}$ siècle, des opinions arrêtées sur les principes théoriques de la restauration ${ }^{130}$, en Bourgogne, Viollet-le-Duc doit tout inventer et notamment les règles matérielles et techniques de celle-ci. Dans ce contexte la diversité des inclinations est légitime; néanmoins, sur une aussi longue durée - plus de vingt ans - cette permanence ne laisse pas d'étonner.

\section{NOTES}

1. * Mes sincères remerciements à $\mathrm{M}^{\text {lle }}$ Delphine Hanquiez.

1. Viollet-le-Duc, cat. expo., Galeries nationales du Grand Palais, Paris, RMN, 1980, 415 p. JeanMichel Leniaud, Viollet-le-Duc ou les délires du système, Paris, Mengès, 1994, 225 p. Viollet-le-Duc à Pierrefonds et dans l'Oise, Actes du colloque international du château de Pierrefonds, le 6 et 7 juin 2007, dir. Jean-Paul Midant, Paris, Monum', coll. Idées et Débats, 2008.

2. 2. Laurent Baridon, L'Imaginaire scientifique de Viollet-le-Duc, Paris, L'Harmattan, 1996, 293 p.

3. 3. Les mémoires suivants de $2^{\mathrm{e}}$ cycle de l'École du Louvre ont été réalisés sous la direction d'Isabelle Pallot-Frossard avec notre collaboration et celle de Lise Leroux: Antoine Quesnoit, L'église Sainte-Marie Madeleine à Vézelay: identification des pierres employées lors de la restauration de l'édifice entre 1840 et 1859, 2006; Christine Bongart, La pierre dans le chantier de reconstruction du château de Pierrefonds par Viollet-le-Duc, de 1858 à 1879, 2008; Marie-Clémentine Bonnin, Les matériaux du chantier chez E. Viollet-le-Duc. La pierre sur le chantier de restauration de l'ancienne abbatiale de Saint-Denis, 2008; Nicolas Burette, Les matériaux dans le chantier de construction de la sacristie de la cathédrale de Paris, 2008 ; Marie Derouette, La pierre dans le chantier de restauration de Viollet-le-Duc à la cathédrale Notre-Dame d'Amiens, 2008 ; Pauline Léonet, La pierre dans le chantier de construction de l'église Saint-Denis-de-l'Estrée par Viollet-le-Duc, 1860 à 1866, 2008 ; Nicolas Navarro, Les matériaux dans le chantier d'achèvement de la cathédrale de Clermont-Ferrand, 2008 ; Cathie Paumier, La polychromie au château de Pierrefonds : analyse formelle et interprétation matérielle, 2008.

4. 4. Viollet-le-Duc a également construit une chapelle, détruite durant les années 1960, à Semuren-Auxois.

5. 5. Pour le détail de ces derniers, il convient de consulter les correspondances de l'architecte avec François-Nicolas Comynet et Émile Amé à qui il annonce ces voyages. Arnaud Timbert, Viollet-le-Duc: le chantier de restauration de la Madeleine de Vézelay. Correspondance (1840-1841), Auxerre, SFAY, 2005. Paris, Arch. Pat., s. c. ; Arnaud Timbert, Delphine Hanquiez, Viollet-le-Duc en Bourgogne: correspondances et documents, 2008, recueil tapuscrit. Ces textes constituent un complément aux frais de déplacements fournis à l'administration: Paris, Archives de la Médiathèque de l'architecture et du patrimoine (Arch. Pat.) : $4^{\circ}$ DOC 330.

6. 6. Voir notamment les Rapports sur Saint-Florentin (1842), Saint-Lazare d'Avallon (1843), Pontigny (1845) et Saint-Eusèbe d'Auxerre (1847).

7. 7. Paris, Arch. Pat., $81 / 21 / 442 / 1$.

8. 8. Paris, Arch. Pat., 81/21/462/1. Anne Prache, "Notre-Dame de Semur-en-Auxois ", Congrès Archéologique de France, Auxois-Châtillonnais, 1986, p. 291-292.

9. 9. Paris, Arch. Pat., $81 / 21 / 58$. 
10. 10. Paris, Arch. Pat., 81/21/50/1. Éliane Vergnolle, et al., La collégiale Notre-Dame de Beaune (Côte-d'Or), Paris, éd. du Patrimoine, 1997, p. 7-8.

11. 11. Paris, Arch. Pat., 81/89/212/1. Lydwine Saulnier, « Les vitraux de Saint-Julien-du-Sault », Viollet-le-Duc dans l'Yonne, Auxerre, Archives départementales de l'Yonne, 1980, p. 49-53.

12. 12. Ulrich Knop de Oppeln, Histoire de la restauration du chœur de la cathédrale Saint-Etienne d'Auxerre, thèse doct., dir. Dieter Kimpel, Univ. Stuttgart, 2003, p. 137.

13. 13. Paris, Arch. Pat., 81/89/15. Claude Hohl, «La préfecture de l'Yonne. Ancien évêché d'Auxerre ", Viollet-le-Duc dans l'Yonne, op. cit., p. 45-46. Juliette Didierjean, Patrice Wahlen, De la demeure épiscopale à la préfecture de l'Yonne. L'ancien palais des évêques d'Auxerre et son quartier, Centre d'études médiévales d'Auxerre, CNRS, 3, 1999, p. 56-57.

14. 14. Paris, Arch. Pat., 81/21/436/1. Christian Freigang, Peter Kurmann, «L'église de l'ancien prieuré de Saint-Thibault-en-Auxois: sa chronologie, ses restaurations, sa place dans l'architecture gothique ", Congrès archéologique de France, Auxois-Châtillonnais, Paris, SFA, 1986, p. 276.

15. 15. Paris, Arch. Pat., 81/89/223. Lydwine Saulnier, "L'église de Saint-Père-sous-Vézelay ", Viollet-le-Duc dans l'Yonne, op. cit., p. 21-22.

16. 16. Paris, Arch. Pat., 81/89/38. Claude Hohl, «La collégiale de Montréal », Viollet-le-Duc dans l'Yonne, op. cit., p. 31-32.

17. ${ }^{17}$. Paris, Arch. Pat., $81 / 71 / 32$.

18. 18. Paris, Arch. Pat., $81 / 89 / 236 / 1$. Lydwine Saulnier, "Sens: la restauration du palais synodal », Viollet-le-Duc dans l'Yonne, op. cit., p. 66-71.

19. 19. Arnaud Timbert, Francesca Lupo, «L'église d'Aillant-sur-Tholon : œuvre d'E.-E. Viollet-leDuc ", L'architecture gothique à Auxerre et dans sa région, Actes de la journée d'études d'Auxerre, 17 mai 2008, dir. Arnaud Timbert, Auxerre, SFAY, 2009, à paraître.

20. 20. Arnaud Timbert, Viollet-le-Duc: le chantier de restauration de la Madeleine de Vézelay, op. cit., 2005.

21. 21. Olivier Liardet, "Émile Amé ", Notre-Dame de La Treille. Du rêve à la réalité, histoire de la cathédrale de Lille, Lille, éd. Yris, 2002, p. 237-238.

22. 22. Auxerre, Bib. mun., Fonds Émile Amé, 188 lettres, s. c. Pour transcription : Arch. Pat. : s. c. Arnaud Timbert, Delphine Hanquiez, Viollet-le-Duc en Bourgogne : correspondances et documents, op. cit., 2008.

23. 23. Aillant-sur-Tholon: Arch. mun., s. c. Voir également la correspondance conservée à Auxerre : Arch. dép. de l'Yonne, 20151.

24. 24. Arnaud Timbert, Francesca Lupo, « L'église d'Aillant-sur-Tholon : œuvre d'E.-E. Viollet-leDuc ", op. cit.

25. 25. Arch. Pat., s. c. Arnaud Timbert, Delphine Hanquiez, Viollet-le-Duc en Bourgogne: correspondances et documents, op. cit., 2008.

26. 26. Il reviendra par la suite sur cette identification au profit des carrières de Coutarnoux. Eugène-Emmanuel Viollet-le-Duc, Dictionnaire raisonné de l'architecture française $d u X^{e} a u X^{e} I^{e}$ siècle , Ligugé, 1997, t. II, p. 278, art. « Carrière».

27. 27. Paris, Arch. Pat., 81/89/300, carton 85, Rapport sur l'État actuel de l'ancienne église de La Madeleine de Vézelay (Yonne) et sur les réparations à faire à cet édifice, E. Viollet-le-Duc, 21 mars 1840.

28. 28. Il faut en effet admettre, contrairement à des propos anciens, que la capacité de Viollet-le-Duc à déterminer la nature géologique des matériaux est limitée, tout du moins dans ces années 1840, comme l'indique son observation des voûtes de la nef de la Madeleine de Vézelay : «Un fait curieux s'est présenté lors de la destruction des voûtes d'arêtes du onzième siècle. Ces voûtes sont construites en pierre factice composée d'un mélange de chaux, de débris calcaires pulvérisés, et de bruyères. La matière ainsi triturée a été jetée dans des moules en forme de claveaux, puis cuite au four ce qui a détruit la 
partie végétale et n'a laissé qu'une concrétion légère et friable, ayant la forme de moellons de cinquante centimètres carrés environ sur vingt centimètres d'épaisseur environ. C'est en voyant cette construction que j'ai pu seulement m'expliquer comment dans l'état où elles étaient ces voûtes ne s'étaient pas écroulées. " Paris, Arch. Pat. 81/89/300, carton 85 : Lettre de Viollet-le-Duc au ministre de l'Intérieur, juillet 1841. Il apparait qu'ici l'architecte n'a pas reconnu le tuf de rivière, sorte de travertin très poreux qui n'est pas étranger à la région et dans lequel figurent des végétaux à l'état de fossiles. Identification réalisée par Lise Leroux, LRMH. Ces pierres constituaient, comme l'indiquent les prospections récentes effectuées sur l'extrados de la première voûte de la nef, le comblement des voûtains. Francesca Lupo, Les techniques de voûtement dans les monuments bâtis par Eugène Viollet-le-Duc, thèse doct., dir. Rosalba Ientile, univ. Polytechnique de Turin (Italie) et Arnaud Timbert, univ. Charles-de-Gaulle-Lille 3, 2009.

29. 29. Cette réalité est donc contraire aux propos d'Adolphe Guillon, «Sigles ou marques de tâcherons tailleurs de pierre ", Bulletin de la Société des sciences historiques et naturelles de l'Yonne, 1892, p. 500.

30. 30. Paris, Arch. Pat., $81 / 71 / 32$ : Porte Saint-André à Autun. Projet de consolidation, E. Viollet-leDuc, s. d., [1844].

31. 31. Christine Bongart, La pierre dans le chantier de reconstruction du château de Pierrefonds, op. cit., I, p. 30-31.

32. 32. Paris, Arch. Pat., $81 / 89 / 302 / 2$, carton 86 , église de Vézelay, Décompte des travaux exécutés et fournitures faites par le S. Demay, entrepreneur à Vézelay en conformité de son engagement en date du 19 Juillet 1840, 15 novembre 1855, É. Amé. Détail des outils employés avec « taille taillandée » et « taille layée ». Taillandée pour la pierre dense de la Mance et layée pour la pierre tendre de Coutarnoux. La même distinction s'observe à Aillant-surTholon. Arnaud Timbert, Francesca Lupo, «L'église d'Aillant-sur-Tholon: œuvre d'E.-E. Viollet-le-Duc », op. cit.

33. 33. Auxerre, biblio. mun., fonds E. Amé : lettre $n^{\circ} 43,13$ décembre 1847.

34. 34. Ibid., lettre $\mathrm{n}^{\circ} 36,1^{\mathrm{er}}$ septembre 1846 .

35. 35. La même démarche peut être relevée à Amiens, sur le chantier de la cathédrale, Viollet-leDuc refuse la boucharde et sollicite une taille en rapport avec l'époque du monument savoir «à la grosse bretture et layées avec une grande précision ». Marie Derouette, La pierre dans le chantier de restauration de Viollet-le-Duc à la cathédrale Notre-Dame d'Amiens, op. cit., 2008, vol. 1, p. 40.

36. 36. Cette rigueur dans le choix des outils a néanmoins des limites. Pour la réalisation du massif de façade de la cathédrale de Clermont-Ferrand il n'hésite pas à rejeter l'emploi de la boucharde sur les faces cachées du monument - cage d'escalier notamment - alors que cet outil appartient à la technologie médiévale, au profit de la patente, d'invention moderne. Murielle Jenzer, «La boucharde : un outil de la fin du Moyen Âge. L'exemple de l'ancienne église abbatiale de Saint-Claude ", Bulletin monumental, 1998, p. 341-353. JeanClaude Bessac, «La patente ", L'Outillage traditionnel du tailleur de pierre de l'Antiquité à nos jours, Paris, CNRS, 1993, p. 87-91.

37. 37. Arnaud Timbert, op. cit., 2005, lettre $\mathrm{n}^{\circ}$ 43, 21 avril 1841, p. 73.

38. 38. Sur le cloitre, Suzann Schlesinger, « Gotische Romanik - romanische Gotik? Viollet-le-Duc rekonstruiert den Kreuzgang in Vézelay ", Aspekte von Raum und Zeit in der Kunst vom Mittelalter bis zur Gegenwart, dir. N. Hille, Regensburg, Schnell \& Steiner, 2007, p. 173-192.

39. 39. Commune de Chevroches à proximité de Dornecy et de la Mance. Cette pierre fut également employée pour la réfection des baies, des arcs et du dallage de la crypte de la 
cathédrale d'Auxerre. Ulrich Knop de Oppeln, Histoire de la restauration du chœur de la cathédrale Saint-Étienne d'Auxerre, op. cit., 2003, p. 137.

40. 40. Arnaud Timbert, Francesca Lupo, « L'église d'Aillant-sur-Tholon : œuvre d'E.-E. Viollet-leDuc », op. cit.

41. 41. Paris, Arch. Pat., 81/89/236/1 : Ancienne salle synodale de l'archevêché de Sens. Devis des travaux de restauration à entreprendre dans cet édifice, E. Viollet-le-Duc, Paris, 20 février 1851, fo 10 v., fo 25 r., fo 33 r.

42. 42. L'abbatiale de Pontigny présente, dès le XII ${ }^{\mathrm{e}}$ siècle, la même répartition entre un calcaire à oolithes et entroques (Bathonien) pour les soubassement et un calcaire blanc crayeux du type pierre de Tonnerre (Kimméridgien) pour les parties supérieures des parois et des supports. Annie Blanc, "Les pierres de l'abbaye de Pontigny : recherches des carrières d'origine ", Les cisterciens dans l'Yonne, dir. Terryl N. Kinder, Pontigny, Les amis de Pontigny éd., 1999, p. 98.

43. 43. Antoine Quesnoit, L'église Sainte-Marie Madeleine à Vézelay, op. cit., 2007, p. 23-24.

44. 44. Plaque de faible épaisseur détachée de la couche supérieure d'un banc de roche calcaire du jurassique au nord-ouest de l'Yonne. Benoît Delarozière, « Lave et laviers de Bourgogne ", Lithiques, $\mathrm{n}^{\circ} 6,1989, \mathrm{p} .22$.

45. 45. Calcaire oolithique vacuolaire blanchâtre et blanc jaunâtre à grain moyen. Il se trouve dans le Bathonien supérieur et moyen.

46. 46. Calcaire du Jurassique, type oolithique un peu crayeux, blanc à grains fins.

47. 47. Commune de Thizy, vers Coutarnoux. Calcaire à entroques de teinte café au lait très clair, bleu dans les bancs inférieurs, terrain jurassique, oolithe inférieure.

48. 48. Le dallage de la nef a été refait en 1859, selon le mémoire de l'entrepreneur, en pierre de Thizy pour les travées 1 à 9 , en pierre de la Mance pour la $10^{\mathrm{e}}$ travée proche du chœur et en vieilles pierres pour les bas-côtés.

49. 49. Paris, Arch. Pat., 81/21/436/1, Mémoire des travaux d'art exécutés à l'église de Saint-Thibault pendant l'année 1844 par Marion, entrepreneur, Paris, 15 septembre 1845, art. $2^{\mathrm{e}}$.

50. 50. Paris, Arch. Pat., $81 / 21 / 50 / 1$, Devis de travaux de consolidation et de restauration, Viollet-leDuc, 20 décembre $1844, \mathrm{f}^{\circ} \mathrm{v}$.

51. 51. Paris, Arch. Pat., $81 / 71 / 32$, Porte Saint-André d'Autun. Décompte supplémentaire des travaux exécutés par Demay, entrepreneur, pendant l'exercice 1848. É. Amé, 20 juillet 1849, fo $1 \mathrm{v}$.

52. 52. Paris, Arch. Pat., 81/89/152, Église de Montréal. Devis des travaux à exécuter pour consolider l'édifice, Paris, le 31 octobre 1844, E. Viollet-le-Duc, fo 1v. Église de Montréal, Rapport d'E. Viollet-leDuc, le 25 janvier $1851, f^{\circ} 8 \mathrm{v}$.

53. 53. Paris, Arch. Pat., $81 / 89 / 15$, Devis descriptif de la restauration de la galerie byzantine ainsi que de la terrasse à construire au-dessus des bureaux de la préfecture, dressé par Boivin, architecte départemental de l'Yonne et signé par E. Viollet-le-Duc, 2 mai 1845, fo 1 r. Pour complément: Auxerre, Arch. dép. de l'Yonne : 74 T 8.

54. 54. Paris, Arch. Pat., 81/89/222, Mémoire supplémentaire des travaux exécutés pendant l'Exercice 1842 à l'église de Saint-Père (Yonne) par Demay, entrepreneur, E. Viollet-le-Duc, 22 Juin 1842 , fo 4 r. et fo 5 r.

55. 55. Paris, Arch. Pat., 81/89/152, Église de Montréal. Rapport d'E. Viollet-le-Duc, le 4 juin 1852, fo 3 r.

56. 56. Paris, Arch. Pat., 81/89/236/1, Ancienne salle synodale de l'archevêché de Sens. Devis des travaux de restauration à entreprendre dans cet édifice, E. Viollet-le-Duc, Paris, 20 février 1851, fo $87 \mathrm{r}$. et $\mathrm{f}^{\circ} 103 \mathrm{r}$.

57. 57. Paris, Arch. Pat., 81/21/462/1. Pour le dallage : Mémoire des ouvrages exécutés à l'église NotreDame de Semur pendant l'année 1851 par Marion, entrepreneur, E. Viollet-le-Duc, 1852, fo 1 v. Pour les 
contreforts : Mémoire des travaux exécutés à l'église Notre-Dame de Semur par Marion, entrepreneur, année 1843, E. Viollet-le-Duc, Semur, le 16 août $1844, f^{\circ} 1$ v. Pour la flèche : Mémoire des ouvrages exécutés par Marion, année 1845, pour la réparation de la flèche de l'église Notre-Dame de Semur, $\mathrm{E}$. Viollet-le-Duc, Semur, le 15 décembre 1845, fo 2 r.

58. 58. Arnaud Timbert, Francesca Lupo, «L'église d'Aillant-sur-Tholon : œuvre d'E.-E. Viollet-leDuc », op. cit.

59. 59. Aillant-sur-Tholon, Arch. mun., s. c., Église d'Aillant-sur-Tholon. Devis supplémentaire pour le dallage de l'église. E. Viollet-le-Duc, Paris, 22 février 1866.

60. 60. Pauline Léonet, op. cit., 2008, vol. 1, p. 22. Christine Bongart, La pierre dans le chantier de reconstruction du château de Pierrefonds, op. cit., I, p. 27-30.

61. 61. Il utilise la pierre de Chastillon-sur-Seine pour les soubassements des portails de la façade occidentale de la cathédrale Notre-Dame de Paris et de sa sacristie. Nicolas Burette, Les matériaux dans le chantier de construction de la sacristie de la cathédrale de Paris, op. cit., 2008, vol. 1, p. 35.

62. 62. Eugène-Emmanuel Viollet-le-Duc, Dictionnaire raisonné..., op. cit., 1997, art. : «Construction», t. IV, p. 128. On pourrait tout autant attribuer à la restauration son propos sur la construction: "Notre temps seul possède des chemins de fer, des engins à vapeur, des moyens d'une énergie et d'une puissance supérieures. Pourquoi donc alors construire comme on le faisait pendant le dernier siècle, particulièrement lorsqu'il s'agit de maçonnerie? » Entretiens sur l'Architecture, Paris, Mardaga, 1986, «12 Entretien », p. 60.

63. 63. Georges Cotteau, «Note sur la provenance géologique des pierre de l'église de la Madeleine de Vézelay", Bulletin de la Société des sciences historiques et naturelles de l'Yonne, t. 18, 1864, p. 156-157. Sur cette pierre, pour complément, voir Pierre Rat, Terroirs et Monuments de France, 1992, p. 45.

64. 64. Commune de Dornecy, entre Vézelay et Clamecy.

65. 65. Arnaud Timbert, Viollet-le-Duc: le chantier de restauration de la Madeleine de Vézelay, op. cit., lettre $n^{\circ} 17,19$ juillet 1840, p. 37.

66. 66. Ibid., lettre $\mathrm{n}^{\circ} \mathrm{XXVI}, 28$ mai 1841 p. 88.

67. 67. Ibid., lettre $\mathrm{n}^{\circ} 12,7$ juillet $1840, \mathrm{p} .31$.

68. 68. Dès le début du chantier, les questions de sculpture préoccupent l'architecte. Il a été admis que Viollet-le-Duc aurait renoncé à la pierre de Courson pour la sculpture et employé celle de Mailly-la-Ville. Lydwine Saulnier, Neil Stratford, La Sculpture oubliée de Vézelay, Paris-Genève, SFA, 1984, p. 18. Cette affirmation n'est pas compatible avec les attachement figurés et les mémoires: la pierre de Mailly n'est jamais citée dans ces derniers, ni dans les dessins annotés de la main de l'architecte précisant des choix de pierre, ni dans les devis à la seule exception de « Deux demi figures d'apôtres détruits à remplacer en pierre de Mailly-la-Ville ... la tête du Christ supérieur et le buste à replacer en pierre de Mailly-la-Ville ", mais cette partie du devis de 1850 ne sera pas réalisée. Selon L. Saulnier et N. Stratford, le tympan central est en pierre de Mailly-la-Ville. Cependant, on doit noter que le code couleur de l'attachement figuré (Auxerre, Arch. dép. de l'Yonne, 2 F 335) identifie la seule pierre de Coutarnoux et qu'un important volume de cette pierre pour la porte centrale de la façade $\left(30 \mathrm{~m}^{3}\right)$ est porté au mémoire de 1852 . Enfin, la correspondance d'Émile Amé précise, le 6 avril 1851, que « le tympan et les deux voussures ont été demandés à Coutarnoux ». Pour plus de détails sur cette question, voir Antoine Quesnoit, L'église Sainte-Marie Madeleine à Vézelay, op. cit., 2007, p. 28.

69. 69. Arnaud Timbert, Viollet-le-Duc: le chantier de restauration de la Madeleine de Vézelay, op. cit., lettre $\mathrm{n}^{\circ} 17,19$ juillet 1840 , p. 38 .

70. 70. Ibid., lettre $\mathrm{n}^{\circ}$ VII, 24 juillet 1840, p. 39. 
71. 71. Robert Vassas, "Travaux à la Madeleine de Vézelay, voûtes de la nef", Monuments historiques de la France, vol. XIV, n 1, 1968, p. 56-61.

72. 72. Benoît Delarozière, op. cit., 1989, p. 29. Le principal inconvénient de ce matériau est le poids. Celui-ci varie, en effet, de 500 à $600 \mathrm{~kg} \mathrm{au} \mathrm{m}^{2}$ et oblige la mise en œuvre de charpentes très fortes. Autre inconvénient, son prix de revient. Dès le XIX ${ }^{\mathrm{e}}$ siècle il semble déjà élevé ce qui n'était pas le cas au Moyen Âge durant lequel la lave était arrachée dans les lavières locales accessibles par le droit d'usage.

73. 73. Paris, Arch. Pat., 81/89/152, Rapport sur l'église de Montréal, E. Viollet-le-Duc, 20 juin 1844, f ${ }^{\circ} 2 \mathrm{r}$.

74. 74. Paris, Arch. Pat., 81/21/58, Mémoire des dépenses faites à l'église de Flavigny, année 1845, par Marion, entrepreneur, Semur, le 25 décembre 1845, E. Viollet-le-Duc, Paris, le 31 décembre 1845, art. $1, \mathrm{f}^{\circ} 1 \mathrm{v}$.

75. 75. Paris, Arch. Pat., 81/89/223, Lettre d'E. Viollet-le-Duc au vicomte de Bondy, préfet de l'Yonne, Paris, le 28 mars 1843.

76. 76. Paris, Arch. Pat., 81/21/50/01, Rapport. Église Notre-Dame de Beaune (Côte-d'Or). Projet de consolidation et de restauration, Viollet-le-Duc, 20 décembre 1844, fo 7 v.: "Les combles qui couvrent les bas-côtés de la nef et leurs chapelle [sic] peuvent être conservés, ils sont couverts en moellons délités appelés lave dans le pays. Cette lourde et misérable couverture laisse passer les eaux de toutes parts.»

77. 77. Anne-Françoise Garçon, Mine et métal. 1780-1880, les nons-ferreux et l'industrialisation, Rennes, PUR, 1998, p. 87-109.

78. 78. Pierre Lebouteux, "Matériaux et formes: l'évolution de l'emploi du métal dans la couverture ", Les Couvertures métalliques, Actes du colloque de Paris, 4 et 5 décembre 1997, Les Cahiers de la section française de l'ICOMOS, $\mathrm{n}^{\circ}$ 19, 1997, p. 12.

79. 79. Françoise Bercé, Les Premiers travaux de la Commission des Monuments historiques (1837-1848), Paris, Picard, 1979, p. 88. Procès verbal de la séance du 26 août 1840.

80. 80. Nicolas Navarro, Les matériaux dans le chantier d'achèvement de la cathédrale de ClermontFerrand, op. cit., I, p. 41.

81. 81. Eugène-Emmanuel Viollet-le-Duc, Entretiens sur l'Architecture, op. cit., « $11^{\mathrm{e}}$ Entretien », p. 31.

82. 82. Paris, Arch. Pat., $81 / 89 / 236 / 1$, Ancienne salle synodale de l'archevêché de Sens. Devis des travaux de restauration à entreprendre dans cet édifice, E. Viollet-le-Duc, Paris, 20 février 1851, fo 116 v.

83. 83. Elena Carmignani, «Le Rapport Duban en 1866 : les moyens de construire les églises avec économie ", L'Architecture religieuse au XIXe siècle. Entre éclectisme et rationalisme, dir. Bruno Foucart et Françoise Hamon, Paris, PUPS, 2006, p. 234. Ce dossier a déjà été étudié par Jean-Michel Leniaud, dans "Architecture et prix de revient », rééd. dans La Révolution des signes. L'art à l'église, 1830-1930, Paris, éd. du cerf, 2008.

84. 84. Claude Hohl, "L'église de Saint-Florentin ", Viollet-le-Duc dans l'Yonne, Auxerre, 1980, p. 26-27. Viollet-le-Duc s'exprima par ailleurs clairement sur le choix de ce matériau pour les arcs-boutants sous combles d'Aillant-sur-Tholon en estimant que «cette disposition [...] permet d'éviter les arcs-boutants extérieurs, lesquels ne peuvent être construits qu'à grand frais à l'aide de matériaux de choix, et qui a surtout [la brique] l'avantage d'influer sur l'économie de l'édifice». Arnaud Timbert, Francesca Lupo, « L'église d'Aillant-sur-Tholon : œuvre d'E.-E. Viollet-le-Duc », op. cit.

85. 85. Paris, Arch. Pat., 81/89/236/1, Ancienne salle synodale de l'Archevêché de Sens. Devis des travaux de restauration à entreprendre dans cet édifice, E. Viollet-le-Duc, Paris, 20 février 1851, fo 102 v. La même technique sera employée pour la couverture des chapelles orientales de la cathédrale 
d'Amiens. Marie Derouette, La pierre dans le chantier de restauration de Viollet-le-Duc à la cathédrale Notre-Dame d'Amiens, op. cit., I, p. 45.

86. 86. Ibid., 2008, vol. 1, p. 39. Marie-Clémentine Bonnin, Les matériaux du chantier chez E. Viollet-leDuc. La pierre sur le chantier de restauration de l'ancienne abbatiale de Saint-Denis, op. cit., I, p. 16.

87. 87. Marie Derouette, La pierre dans le chantier de restauration de Viollet-le-Duc à la cathédrale Notre-Dame d'Amiens, op. cit., I, p. 39. Christine Bongart, La pierre dans le chantier de reconstruction du château de Pierrefonds, op. cit., I, p. 48.

88. 88. Nicolas Burette, Les matériaux dans le chantier de construction de la sacristie de la cathédrale de Paris, op. cit., I, p. 42.

89. 89. Eugène-Emmanuel Viollet-le-Duc, « Première apparition de Villard de Honnecourt, architecte du XIII ${ }^{\mathrm{e}}$ siècle ", Gazette des Beaux-Arts, t. II, 1859, p. 294. Jean-Michel Leniaud, Les Bâtisseurs d'avenir. Portraits d'architectes (XIX ${ }^{e}-X X^{e}$ siècle), Paris, Fayard, 1998, p. 109.

90. 90. Lydwine Saulnier, «Vézelay : la restauration de l'église de la Madeleine », Viollet-le-Duc, op. cit., p. 60.

91. 91. Arnaud Timbert, « Les arcs-boutants de la nef de l'église abbatiale Sainte-Marie-Madeleine de Vézelay ", Annales de Bourgogne, 2002, t. LXXIV, p. 25-38.

92. 92. Paris, Arch. Pat. : $81 / 89 / 302$ et 303.

93. 93. Georges Cotteau, «Note sur la provenance géologique des pierre de l'église de la Madeleine de Vézelay », op. cit., p. 154.

94. 94. Christine Bongart, La pierre dans le chantier de reconstruction du château de Pierrefonds, op. cit., I, p. 38. Duban n'agira pas autrement à Blois, voir Paul Léon, La Vie des monuments français, destruction, restauration, Paris, Picard, 1951, p. 232.

95. 95. Marie Derouette, La pierre dans le chantier de restauration de Viollet-le-Duc à la cathédrale Notre-Dame d'Amiens, op. cit., I, p. 30.

96. 96. Théodore Château, Technologie du bâtiment ou étude complète des matériaux de toute espèce employés dans les constructions depuis leur fondation jusque et y compris leur décoration, Paris, Ducher, 1880 , p. 253.

97. 97. Beauvais, Arch. dép. de l'Oise, 3 V 5 : Cahier des charges générales, 30 août 1858.

98. 98. Auxerre, Bib. mun., Fonds Émile Amé : lettre $n^{\circ} 88,16$ février 1851.

99. 99. Ibid., lettre $\mathrm{n}^{\circ} 78,9$ août 1850 .

100. 100. Antoine Quesnoit, L'église Sainte-Marie Madeleine à Vézelay, op. cit., p. 24.

101. 101. Arnaud Timbert, Viollet-le-Duc : le chantier de restauration de la Madeleine de Vézelay, op. cit., lettre ${ }^{\circ}$ I, 12 mai 1840, p. 19.

102. 102. Ibid., 2005, lettre $n^{\circ} 4,10$ mai 1840 , p. 18 ; lettre $n^{\circ} 5,25$ mai 1840 , p. 20.

103. 103. Ibid., 2005, lettre $n^{\circ} 4,10$ mai 1840, p. 18.

104. 104. Ibid., 2005, lettre $n^{\circ}$ III, 28 mai 1840, p. 23.

105. 105. Paris, Arch. Pat., 81/21/462/1, Mémoire des travaux d'art faits à l'église Notre-Dame de Semur pendant l'année 1844 par Marion, entrepreneur, art. 1. A Semur, le 15 août 1845.

106. 106. À Clermont-Ferrand, Viollet-le-Duc a également privilégié le sapin pour les échafaudages. Nicolas Navarro, Les matériaux dans le chantier d'achèvement de la cathédrale de Clermont-Ferrand, op. cit., I, p. 35.

107. 107. Paris, Arch. Pat., 81/89/152, Devis des travaux à exécuter pour consolider l'édifice, E. Violletle-Duc, Paris, le 31 octobre 1844, fo $3 \mathrm{r}$.

108. 108. Paris, Arch. Pat., 81/21/58, Mémoire des ouvrages faits à l'église de Flavigny pendant l'année 1846 par Marion, entrepreneur, E. Viollet-le-Duc, 3 novembre 1846, fo $1 \mathrm{r}$.

109. 109. Paris, Arch. Pat., $81 / 21 / 50 / 1$, Devis de travaux de consolidation et de restauration, E. Violletle-Duc, 20 décembre 1844 , fo $2 \mathrm{v}$. 
110. 110. Paris, Arch. Pat., 81/89/236/1, Ancienne salle synodale de l'archevêché de Sens. Devis des travaux de restauration à entreprendre dans cet édifice, E. Viollet-le-Duc, Paris, 20 février 1851, fo ${ }^{\circ} 106$, v.

111. 111. Arnaud Timbert, Viollet-le-Duc : le chantier de restauration de la Madeleine de Vézelay, op. cit., lettre $n^{\circ} 2,21$ avril 1840, p. 16-17 ; lettre $n^{\circ}$ 5, 25 mai 1840, p. 20-22.

112. 112. Auxerre, biblio. mun., fonds Émile Amé, lettre $n^{\circ} 12,18$ novembre 1846.

113. 113. Ibid., lettre $\mathrm{n}^{\circ}$ 6, 10 mars 1849 .

114. 114. Ibid., lettre $\mathrm{n}^{\circ} 90$, le dimanche 18 [sic].

115. 115. Paris, Arch. Pat., 81/89/236/1, Ancienne salle synodale de l'archevêché de Sens. Devis des travaux de restauration à entreprendre dans cet édifice, E. Viollet-le-Duc, Paris, 20 février 1851, fo 104 $\mathrm{v}$. et $105 \mathrm{r}$. La location s'étendra également aux cintres et aux étais. Viollet-le-Duc procèdera de même à Pierrefonds, voir Christine Bongart, La pierre dans le chantier de reconstruction du château de Pierrefonds, op. cit., I, p. 26.

116. 116. Arnaud Timbert, Francesca Lupo, «L'église d'Aillant-sur-Tholon : œuvre d'E.-E. Violletle-Duc », op. cit.

117. 117. Paris, Arch. Pat., 81/89/152, Devis des travaux à exécuter pour consolider l'édifice, Viollet-leDuc, Paris, le 31 octobre 1844, fo 2 r. Arnaud Timbert, Viollet-le-Duc: le chantier de restauration de la Madeleine de Vézelay, op. cit., lettre $\mathrm{n}^{\circ} \mathrm{IV}, 5$ juin 1840, p. 26

118. 118. Paris, Arch. Pat, 81/21/462/1, Procès verbal de réception des travaux exécutés à l'église NotreDame de Semur pendant l'année 1847 par Marion, entrepreneur, Semur, E. Viollet-le-Duc, 25 décembre $1847, \mathrm{f}^{\circ} 1 \mathrm{r}$.

119. 119. Paris, Arch. Pat., 81/21/50/1, Rapport. Église Notre-Dame de Beaune. Projet de consolidation et de restauration, E. Viollet-le-Duc, 20 décembre 1844, fo $7 \mathrm{r}$.

120. 120. Jean-Michel Leniaud, Les Bâtisseurs d'avenir, op. cit., p. 114.

121. 121. Voir notamment les restaurations de Cheussey à Amiens : Marie Derouette, La pierre dans le chantier de restauration de Viollet-le-Duc à la cathédrale Notre-Dame d'Amiens, op. cit., I, p. 15.

122. 122. Arnaud Timbert, Viollet-le-Duc : le chantier de restauration de la Madeleine de Vézelay , op. cit., lettre $\mathrm{n}^{\circ} 56,9$ juin 1841 , p. 90.

123. 123 . Ibid., 2005, lettre $n^{\circ} 27,11$ octobre 1840 , p. 50 ; lettre $n^{\circ} 28,21$ octobre 1840, p. 52, lettre $\mathrm{n}^{\circ} 29,22$ octobre 1840, p. 53.

124. 124. Paris, Arch. Pat., $81 / 71 / 32$ : Porte Saint-André à Autun. Projet de consolidation, E. Viollet-leDuc, s. d. [1844], fo 4 v.

125. 125. Paris, Arch. Pat., 81/21/58: Mémoire des travaux d'art exécutés à l'église de Flavigny pendant l'année 1884 par Marion, entrepreneur, E .Viollet-le-Duc, 15 août 1845, fo 1.

126. 126. Paris, Arch. Pat., 81/89/152 : Rapport. Église de Montréal, E. Viollet-le-Duc, Paris, 25 janvier 1851, fo 5 r. Sur cette technique : Mathieu Tricoit, « Abbatiale de Saint-Denis. Liaisonnage au plomb des réseaux du triforium », L'homme et la matière: l'emploi du plomb et du fer dans l'architecture gothique, Actes du colloque de Noyon, 16-17 novembre 2006, dir. Arnaud Timbert, Paris, Picard, sous presse.

127. 127. Paris, Arch. Pat., 81/21/462/1: Mémoire des travaux exécutés à l'église de Semur pendant l'année 1846 par Marion, entrepreneur, E. Viollet-le-Duc, 22 janvier 1847, fo 2 v.

128. 128. Paris, Arch. Pat., 81/21/436/1: Mémoire des travaux d'art exécutés à l'église Saint-Thubault pendant l'année 1844 par Marion, entrepreneur, E. Viollet-le-Duc, Paris, 15 septembre 1845, art. 2.

129. 129. Marie-Clémentine Bonnin, Les matériaux du chantier chez E. Viollet-le-Duc. La pierre sur le chantier de restauration de l'ancienne abbatiale de Saint-Denis, op. cit., I, p. 6.

130. 130. Jean-Michel Leniaud, Jean-Baptiste Lassus (1807-1857) ou le temps retrouvé des cathédrales, Paris, SFA-Droz, 1980, p. 77-82. 


\section{RÉSUMÉS}

Parmi la multiplicité des recherches qui font l'actualité des études sur Eugène-Émmanuel Violletle-Duc, l'analyse des sources inhérentes à l'histoire des chantiers tient une place de premier ordre. Ces documents offrent, d'une part, une matière favorable à une connaissance précise des chantiers de Viollet-le-Duc en Bourgogne, parmi lesquels Vézelay, Montréal, Semur-en-Auxois et, d'autre part, favorisent une approche plus juste de «Viollet-le-Duc maître d'œuvre » en dévoilant une tension entre le théoricien et le praticien homme de la matière. Cet article propose, à travers des archives pour une grande part inédites - dont 190 lettres de Viollet-le-Duc à Émile Amé, second inspecteur des travaux pour Vézelay et la Bourgogne - de présenter les démarches engagées par l'architecte pour l'achat de la pierre; d'apprécier les raisons qui contribuèrent au choix des carrières, des entrepreneurs en maçonnerie mais encore en charpenterie ; d'évaluer les critères qui présidèrent au choix des essences de bois (sapin, chêne) ; enfin, de mesurer la place et le rôle du métal dans la restauration (boulon, liaisonnage au plomb, zinc, etc.).

The analysis of archival sources dealing with the history and the progress of the restoration sites is one of the most important aspects developed in the numerous recent works on EugèneÉmmanuel Viollet-le-Duc. First of all, the study of these documents seems to be the best way to get a precise knowledge of the construction sites of Viollet-le-Duc in Burgundy, like Vézelay, Montréal or Semur-en-Auxois, and on the other hand, it becomes easier to get a right vision of Viollet le Duc as a "maitre d'oeuvre", revealing a tension between the theorist and the practitioner. Thought a lot of unpublished archives (among them 190 letters from Viollet le Duc to Emile Amé, second inspector of works for the site of Vézelay and all the Burgundy) this paper aims to present the steps that engaged the architect for the buying of the stone, how and for what reasons he chose the quarries, the stoneworking and carpenting contractors, the sorts of wood (fir and oak), and at last to define the place and the part of the metal in restoration (bolts, lead, zinc, etc...)

Unter den vielfältigen aktuellen Studien über Eugène-Emmanuel Viollet-le-Duc spielen die Untersuchungen von Quellen, die sich auf die Geschichte der Baustellen beziehen, eine erstrangige Rolle. Solche Dokumente bieten einerseits Stoff für eine genauere Kenntnis der Baustellen Viollet-le-Ducs in Burgund, darunter Vézelay, Montréal und Semur-en-Auxois ; sie sind andererseits besonders repräsentativ für die Tätigkeit Viollet-le-Ducs als leitender Architekt, indem sie eine gewisse Spannung zwischen dem Theoretiker und dem Praktiker anschaulich machen. Anhand von größtenteils unveröffentlichen Archiven - darunter 190 Briefe Viollet-le-Ducs an Emile Amé, zweiter Bauinspektor für Vézelay und Burgund - nimmt sich dieser Artikel vor, das Vorgehen des Architekten beim Einkauf des Steines zu verfolgen; seine Kriterien zur Auswahl der Steinbrüche, der Maurer- oder Zimmererunternehmer, der Holzarten (Kiefer, Eiche) darzulegen und die bedeutsame Rolle des Metalls in den Restaurierungsarbeiten (Bolzen, Bleiverbindungen, Zink usw.) herauszustellen. 


\section{AUTEUR}

\section{ARNAUD TIMBERT}

Arnaud Timbert, après une thèse de doctorat sur « Le chevet de La Madeleine de Vézelay et le début de l'architecture gothique en Bourgogne » soutenue sous la direction d'Éliane Vergnolle, a mené des recherches sur la cathédrale Notre-Dame de Noyon dans le cadre d'un programme pluridisciplinaire engagé en 2002. Il est actuellement maître de conférences à l'université Charles-de-Gaulle-Lille 3 et co-responsable de l'équipe de recherche $n^{\circ} 1$ de l'IRHiS-UMR 8529 . 